\title{
Genome and Fatty Acid Analysis of Pseudomonas stutzeri
}

\author{
PAUL B. RAINEY, ${ }^{1 *}$ IAN P. THOMPSON,${ }^{1}$, AND NORBERTO J. PALLERONI ${ }^{2}$ \\ Molecular Microbial Ecology, Institute of Virology and Environmental Microbiology, Oxford OX1 3SR, \\ United Kingdom, ${ }^{1}$ and Department of Microbiology, New York University Medical Center, New York,
} New York $10016^{2}$

\begin{abstract}
A genome and fatty acid analysis of 16 Pseudomonas stutzeri reference strains having DNA compositions ranging from 62.2 to $65.5 \mathrm{~mol} \% \mathrm{G}+\mathrm{C}$ was performed by pulsed-field gel electrophoresis of $\mathrm{XbaI}$ and SpeI macrorestriction fragments and gas chromatography of total cellular fatty acids. Macrorestriction fragment patterns were evaluated by using previously described algorithms (D. Grothues and B. Tümmler, Mol. Microbiol. 5:2763-2776, 1991), and the results allowed us to subdivide the species into two groups which correlated with $\mathbf{G}+\mathbf{C}$ content. Two examples of recent strain divergence were observed among clinical isolates, but in general a marked degree of heterogeneity was observed in the macrorestriction fragment patterns, and even phenotypically similar strains produced divergent patterns. While the differences were not sufficiently great to exclude any strain from $P$. stutzeri, they suggest that recombination and niche-specific selection may be significant factors responsible for generating and maintaining the heterogeneity inherent in the species. Genome sizes were estimated from the sums of SpeI restriction fragment sizes and ranged from 3.4 to 4.3 Mbp; the genome sizes of the low-G+C-content strains $(G+C$ contents, approximately $62 \mathrm{~mol} \%$ ) were confined to a narrow range between 3.9 and 4.1 Mbp. An examination of the distributions of macrorestriction fragments resulting from digestion with $\mathrm{XbaI}$ and $\mathrm{SpeI}$ showed that both distributions differed significantly from the expected (random) distribution, suggesting that there is a supragenic level of chromosomal organization. An analysis of fatty acid methyl ester data by using Microbial Identification System software revealed a similar correlation between phenotype and $\mathrm{G}+\mathrm{C}$ content, indicating that division of the species is possible by the method used in this study. For comparative purposes, a numerical analysis of previously reported substrate utilization data (N. J. Palleroni, M. Doudoroff, R. Y. Stanier, R. E. Solánes, and M. Mandel, J. Gen. Microbiol. 60:215-231, 1970) was performed. The results of this analysis revealed that there was a relationship among strains which showed no correlation with the results obtained from either the macrorestriction fragment analysis or the fatty acid methyl ester analysis.
\end{abstract}

Pseudomonas stutzeri is a nonfluorescent denitrifying pseudomonad which is commonly encountered in diverse habitats, including soil, water, and sewage (12); it is also associated with a variety of blood, respiratory, urinary, and cerebrospinal fluid infections of humans (8). The ability of $P$. stutzeri to reduce nitrate to dinitrogen gas has led to its use as a model system for studying the genetics and biochemistry of denitrification (2). In addition, the metabolic diversity of $P$. stutzeri has resulted in the use of selected strains in bioremediation (3), biological control (7), and the food industry (28).

$P$. stutzeri is a member of Pseudomonas rRNA group I (19) and is distinguished from other pseudomonads on the basis of the wrinkled colony morphology of fresh isolates, the ability to utilize maltose, starch, glycolate, and propylene glycol as sole carbon and energy sources, and the production of dinitrogen gas from nitrate (16). Despite these conserved traits, $P$. stutzeri is characterized by a high degree of phenotypic heterogeneity $(17,18,22,24)$ and a broad range of DNA base compositions (60.6 to $66.3 \mathrm{~mol} \% \mathrm{G}+\mathrm{C})$ $(11,16,18,22)$. This range of DNA base compositions is almost as great as that found in the entire genus Pseudomonas and suggests that subdivision of the species is war-

${ }^{*}$ Corresponding author. Mailing address: Molecular Microbial Ecology, Institute of Virology \& Environmental Microbiology, Mansfield Road, Oxford OX1 3SR, United Kingdom. Phone: (0)865 512361. Fax: (0)865 59962. Electronic mail address: Paul.Raney@ St-Cross.Oxford.ac.uk. ranted. Mandel (11) initially proposed subdivision of this taxon into two species on the basis of DNA base compositions; strains having $\mathrm{G}+\mathrm{C}$ contents of approximately 65 mol\% were assigned to $P$. stutzeri, while strains having $\mathrm{G}+\mathrm{C}$ contents of approximately $62 \mathrm{~mol} \%$ were assigned to a new species, Pseudomonas stanieri.

Subsequent studies performed with a greater selection of strains $(18,22)$ have failed to find any substantive correlation between phenotypic characteristics and nucleic acid base composition, and therefore the distinction between $P$. stutzeri and $P$. stanieri (11) has been abandoned (18). Recent work in which DNA-DNA hybridization techniques have been used has confirmed the presence of distinct genomic groups in the species $(18,22)$, and Rosselló et al. (22) have proposed adoption of the term genomovar to describe genomic groups of nomenspecies. In their analysis of 49 strains, which included 14 strains used in previous studies, Rosselló et al. (22) established seven separate genomovars, three of which corresponded to genomic groups I, II, and IV described by Palleroni et al. (18).

The main purpose of this investigation was to explore the genome structures of $16 P$. stutzeri strains studied in detail by Stanier et al. (24), Mandel (11), and Palleroni et al. (18) in order to determine the degree of variability among strains and to attempt to correlate macrorestriction patterns with known properties that are useful for internal subdivision of the species. Other studies described below include an examination of the fatty acid profiles of these strains and a numerical analysis of substrate utilization profiles obtained from data reported by Palleroni et al. (18). 
TABLE 1. $P$. stutzeri strains used

\begin{tabular}{|c|c|c|c|}
\hline Strain $^{a}$ & Source & $\mathrm{G}+\mathrm{C}$ content $(\mathrm{mol} \%)^{b}$ & Genome size $(\mathrm{Mbp})$ \\
\hline $220(=$ ATCC 17587$)$ & Clinical (Copenhagen, Denmark) & $62.2(62.3)$ & 3.9 \\
\hline $221(=$ ATCC 17588) & Clinical (Copenhagen, Denmark) & $65.2(65.0)$ & 4.2 \\
\hline $222(=$ ATCC 17589$)$ & Clinical (Copenhagen, Denmark) & $64.7(64.5)$ & 3.8 \\
\hline $224(=$ ATCC 17591) & Clinical (Copenhagen, Denmark) & $62.2(61.4)$ & 4.1 \\
\hline $225(=$ ATCC 17592$)$ & Clinical (Copenhagen, Denmark) & $62.7(60.9)$ & 4.1 \\
\hline $226(=$ ATCC 17593$)$ & Clinical (Copenhagen, Denmark) & $64.7(64.1)$ & 4.0 \\
\hline $227(=$ ATCC 17594$)$ & Clinical (Copenhagen, Denmark) & $65.5(63.8)$ & 4.3 \\
\hline $228(=$ ATCC 17595$)$ & Clinical (Copenhagen, Denmark) & $62.7(61.5)$ & 4.0 \\
\hline 275 (= ATCC 17639) & Soil & 64.2 & 3.5 \\
\hline $316(=$ ATCC 17682) & Clinical (Paris, France) & 65.7 & 4.1 \\
\hline $318(=$ ATCC 17684$)$ & Clinical (Paris, France) & 65.2 & 4.2 \\
\hline 319 (= ATCC 17685) & Clinical (Paris, France) & 65.2 & 3.6 \\
\hline $320(=$ ATCC 17686$)$ & Clinical (Paris, France) & 65.2 & 4.0 \\
\hline $321(=$ ATCC 17687) & Clinical (Paris, France) & 65.2 & 4.0 \\
\hline $419(=$ ATCC 17832$)$ & Soil (Berkeley, Calif.) & $65.2(64.9)$ & 3.8 \\
\hline $\mathrm{CH} 88^{c}$ & Water (Mendoza, Argentina) & $64.3(63.6)$ & 3.4 \\
\hline
\end{tabular}

a Strain designations of Stanier et al. (24).

${ }^{b}$ Data from reference 18. The values in parentheses are from reference 22 .

${ }^{c}$ See reference 18 . Strain $\mathrm{CH} 88$ was isolated by using L-tatrate enrichment.

\section{MATERIALS AND METHODS}

Bacterial strains. A total of 16 reference strains were studied (Table 1), and phenotypic properties, including substrate utilization patterns, number and location of flagella, temperature range for growth, biochemical characteristics, and genotypic properties (including DNA base composition and DNA competition data), for all of the strains except strain CH88 have been described previously by Stanier et al. (24) and Mandel (11). Strain CH88 has been described by Palleroni et al. (18), who also reexamined the properties of the other 15 strains included in this study.

DNA preparation. High-quality unsheared DNA was prepared by embedding whole cells in chromosomal grade agarose blocks and then lysing the cells with detergent and proteinase $K(20)$. Cells were grown overnight in LuriaBertani broth, harvested by centrifugation, and washed twice in NET-100 buffer $(0.1 \mathrm{M} \mathrm{NaCl}, 0.1 \mathrm{M}$ EDTA, $0.01 \mathrm{M}$ Tris- $\mathrm{HCl}[\mathrm{pH} 8.0])$. The suspension was mixed with an equal volume of molten $0.9 \%$ chromosomal grade agarose (in NET-100 buffer) and dispensed into molds. The cells were lysed by immersing agarose blocks in lysis solution I $(6 \mathrm{mM}$ Tris- $\mathrm{HCl}$ [pH 7.6], $1 \mathrm{M} \mathrm{NaCl}, 100 \mathrm{mM}$ EDTA [pH 8.0], $0.5 \%$ Sarkosyl, $1 \mathrm{mg}$ of lysozyme per ml) for $24 \mathrm{~h}$ at $37^{\circ} \mathrm{C}$. Lysis solution I was then replaced with lysis solution II $(0.5 \mathrm{M}$ EDTA [pH 9.0], 1\% Sarkosyl, $1.5 \mathrm{mg}$ of proteinase per $\mathrm{ml}$ ), and the preparation was incubated for an additional $48 \mathrm{~h}$ at $50^{\circ} \mathrm{C}$.

Restriction endonuclease digestion. Restriction endonuclease digestions were performed as described previously (26) following phenylmethylsulfonyl fluoride treatment. Digestions were performed in $1 \times$ KGB buffer (12) by using $8 \mathrm{U}$ of enzyme. Each reaction mixture was incubated at $37^{\circ} \mathrm{C}$ for 6 h.

Pulsed-field gel electrophoresis. DNA inserts containing approximately $2 \mu \mathrm{g}$ of DNA were loaded into $0.5 \times \mathrm{TBE}$ buffered gels. The gels were electrophoresed in a contourclamped homogeneous electric field DRII pulsed-field electrophoresis system (Bio-Rad) at $200 \mathrm{~V}$ by using $0.5 \times \mathrm{TBE}$ buffer and were stained with ethidium bromide $\left(0.5 \mu \mathrm{g} \mathrm{ml}^{-1}\right)$ for $30 \mathrm{~min}$. Fragment sizes were estimated by comparison with comigrating ladders of lambda DNA concatemers (BioRad) and EcoRI-HindIII-digested lambda DNA.
Evaluation of pulsed-field gel electrophoresis gels. Enlarged prints of gels were digitized by using a Summagraphics data tablet, and fragment sizes were determined by using Molmatch computer software (UV Products, Cambridge, United Kingdom). The criteria used for evaluating gels have been described previously (6), and a minimum of two gels were prepared per enzyme digest to ensure optimal resolution of all fragments.

Levels of relatedness between strains were determined by conducting comprehensive pairwise comparisons of macrorestriction fragment sizes by using the Dice coefficient $\left(S_{D}\right)$, which is defined as the ratio of twice the number of bands common to each pair of restriction profiles to the total number of macrorestriction fragments in each restriction profile $(6,15): S_{D}=2 n_{i} /\left(n_{A}+m_{B}\right)$, where $n_{A}$ and $m_{B}$ are the numbers of fragments in patterns $A$ and $B$, respectively, and $n_{i}$ is the number of common bands. The statistical significance of pairwise comparisons was evaluated by using equation 13 of Grothues and Tümmler (6), which indicates the confidence interval for the statistical a priori similarity level of macrorestriction fragment profiles:

$$
\left.\left.P\left(S_{D A B}\right)=\underset{K}{2 /\left(\sum n_{K}\right.}+\sum_{K} m_{K}\right) \times \underset{K}{\left[\sum\right.}\left(n_{K} m_{K} / I_{K}\right) \pm t / 2\left(\sum_{K} n_{K}\right)^{1 / 2}\right]
$$

where $K$ is the number of restriction profiles generated by $K$ independent enzyme digestions, $I$ is the number of intervals into which the range of restriction fragments is divided, and $t$ is the value of Student's $t$ distribution (see reference 6 for a detailed description). For both $X b a I$ and SpeI digests, the groups of restriction fragments were divided as follows: 0.5 -kbp intervals between 0 and $15 \mathrm{kbp}$; $1-\mathrm{kbp}$ intervals between 15 and $24 \mathrm{kbp}$; 2-kbp intervals between 25 and 100 kbp; 3-kbp intervals between 101 and $148 \mathrm{kbp}$; 4-kbp intervals between 149 and $199 \mathrm{kbp}$;-kbp intervals between 200 and $299 \mathrm{kbp}$; and 10-kbp intervals for sizes greater than 300 kbp.

Results from this analysis were interpreted as described previously (6). Briefly, an $S_{D}$ value within the calculated confidence interval (for example, 95\%) indicated that the level of similarity between a pair of strains was no more than that expected by chance, given a random distribution of restriction sites and a certain genome size. Such a result indicated that the strains compared belong to the same 
species (strains belonging to the same species have similar DNA base compositions, codon usage characteristics, and genome sizes) (6). An $S_{D}$ value outside the $95 \%$ confidence interval indicated that the strains are either significantly more closely related (if the $S_{D}$ was higher) or less closely related (if the $S_{D}$ was lower) $(P<0.05)$ than expected on the basis of genome sizes and a random distribution of restriction sites. If the $S_{D}$ value was greater than the upper value of the $95 \%$ confidence interval, then the strains compared probably share a common ancestor (and may be assigned to the same biovar). If the $S_{D}$ value was less than the lower value of the $95 \%$ confidence interval, then the strains compared probably do not belong to the same taxospecies.

Cellular fatty acid analysis. Strains were cultured on tryptic soy broth agar for $24 \mathrm{~h}$ at $28^{\circ} \mathrm{C}$, and whole-cell fatty acids were saponified, methylated, and extracted as described previously (14). Fatty acid methyl esters (FAMEs) were analyzed by using a Hewlett-Packard series II gas chromatograph. The names of FAMEs were determined by using Microbial Identification System software, and isolates were identified by using the Microbial Identification System aerobe library (version 3.30). All strains were analyzed in triplicate, and the mean profile was used to generate a unique library for each strain.

Numerical analysis. Levels of similarity between macrorestriction fragment profiles were determined by using the $S_{D}$, and strains were clustered by using the unweighted pair group method using arithmetic averages (UPGMA) (23). Levels of similarity between FAME profiles were calculated by using the generalized similarity coefficient of Gower (5) and the coefficient based on Euclidean distance (23). To facilitate comparisons between the results of this study and the phenotypic properties of the 16 strains described previously, the substrate utilization data of Palleroni et al. (18) (data for 80 variably utilized substrates) were subjected to a numerical analysis. Levels of similarity between substrate utilization profiles were calculated by using the method used for FAME profiles. In all cases clustering of isolates was accomplished by using the UPGMA (23).

\section{RESULTS}

Quantitative evaluation of macrorestriction patterns. Two rarely cutting restriction endonucleases, $\mathrm{XbaI}\left(5^{\prime}\right.$-TCTAGA) and SpeI (5'-ACTAGT), both of which contain the tetranucleotide CTAG in the recognition sequence (13), were used to cleave the $\mathrm{G}+\mathrm{C}$-rich $P$. stutzeri genome and yielded between 33 and 42 and between 24 and 39 fragments, respectively (exclusive of doublets and triplets). Other potentially rarely cutting enzymes, including DraI (5'-TT TAAA), $P a c$ I (5'-TTAATTAA), and $S w a I$ (5'-ATTTAAAT), were used to cleave $P$. stutzeri DNA, but yielded either too few (PacI and SwaI) or too many (DraI) fragments to be useful in pairwise comparisons (data not shown) (4). The size range and distribution of fragments generated by $X b a \mathrm{I}$ and SpeI were optimal for resolution by pulsed-field gel electrophoresis and facilitated accurate assessment of differences and similarities in restriction patterns.

Approximate genome sizes were obtained by adding SpeI restriction fragment sizes (Table 1). The genome sizes ranged from 3.4 to $4.3 \mathrm{Mbp}$, and significantly, all of the strains belonging to the low-G+C-content group of Mandel (11) had genome sizes confined to a narrow band between 3.9 and 4.1 Mbp.

Figure 1 shows the discriminatory value of both $X b a \mathrm{I}$ and SpeI (particularly $X b a \mathrm{I}$ ) when these enzymes were used to cleave DNAs prepared from $P$. stutzeri strains. Visual assessment of the $X b a I$ restriction data revealed two groups which could be distinguished on the basis of the presence or absence of restriction fragments larger than $150 \mathrm{kbp}$. All of the strains lacking fragments of this size (strains 220, 224, $225,228,320$ ) had $\mathrm{G}+\mathrm{C}$ contents between 62.2 and 62.7 mol\% (18).

Distribution of restriction fragments. Figure 2 shows the distribution of both $X b a I$ and SpeI restriction fragments obtained from the $16 P$. stutzeri strains. The theoretical distribution of fragments is shown for comparative purposes and was calculated for both enzymes by using the distribution function: $P(x)=\mu(1-\mu)^{x-1}$, where $\mu$ is the reciprocal of the mean fragment length, (70 kbp for $X b a \mathrm{I}$ and $100 \mathrm{kbp}$ for SpeI) and $x$ is the fragment size (a random distribution of enzyme recognition sites was assumed) (6). In both instances the observed distribution of restriction fragment lengths was significantly different from the theoretical random distribution: $X b a \mathrm{I}, \chi_{[41]}^{2}(P<0.04)$; SpeI, $\chi_{[47]}^{2}(P<0.001)$. This was most noticeable for SpeI, with which significant deviations from expected values occurred throughout the entire range and maxima were detected approximately every 25 to $30 \mathrm{kbp}$. The distribution of $\mathrm{Xba}$ I fragments was more similar to the expected distribution, and the statistically significant $\chi^{2}$ value is explained largely by the presence of three times the expected number of fragments larger than $325 \mathrm{kbp}$.

With the exception of isolates displaying obvious signs of common lineage, no conserved fragments were detected; in fact, a striking feature of the restriction patterns was the substantial amount of between-strain variation. Two obvious examples of common lineage were observed among strains isolated from a common clinical source in Copenhagen, Denmark. Strains 220 and 224 differed by only a single SpeI fragment, and strains 226 and 227 differed by three SpeI fragments and two $\mathrm{XbaI}$ fragments.

Levels of similarity of fragment patterns. The levels of similarity of fragment patterns were determined from pairwise comparisons and were quantified by using $S_{D}(6)$. The confidence intervals for the statistical correspondence of each pair of patterns were calculated by using the equation described above, and this technique was used as a means of discriminating between strains. Approximately one-half of the comparisons shown in Table 2 yielded $S_{D}$ values which were above the upper limit of the $95 \%$ confidence interval, indicating that the levels of similarity for fragment patterns for the pairs of strains were greater than the levels expected on the basis of the oligonucleotide frequency and a random distribution of restriction sites (6). High degrees of relatedness between fragment patterns $(P<0.001)$ were detected among some strains, especially those strains which originated from a common source, indicating that these organisms belonged to a lineage of related strains. No $S_{D}$ values were below the lower limit of the confidence interval, which is consistent with the results of previous work in which relatedness of these bacteria at the species level was demonstrated $(11,18,24)$.

The dendrogram in Fig. 3 shows five clusters at or below the $39 \%$ level of similarity, and one strain, strain 275 , which was not assigned to any cluster. A significant feature of the strains in cluster $I$ is that they all had low $\mathrm{G}+\mathrm{C}$ contents. The relatively low levels of relatedness of all of the restriction patterns, with the exception of those derived from strains which obviously shared common ancestry, are direct reflections of the heterogeneity of the restriction patterns. Nevertheless, despite the differences, all of the macrorestriction 

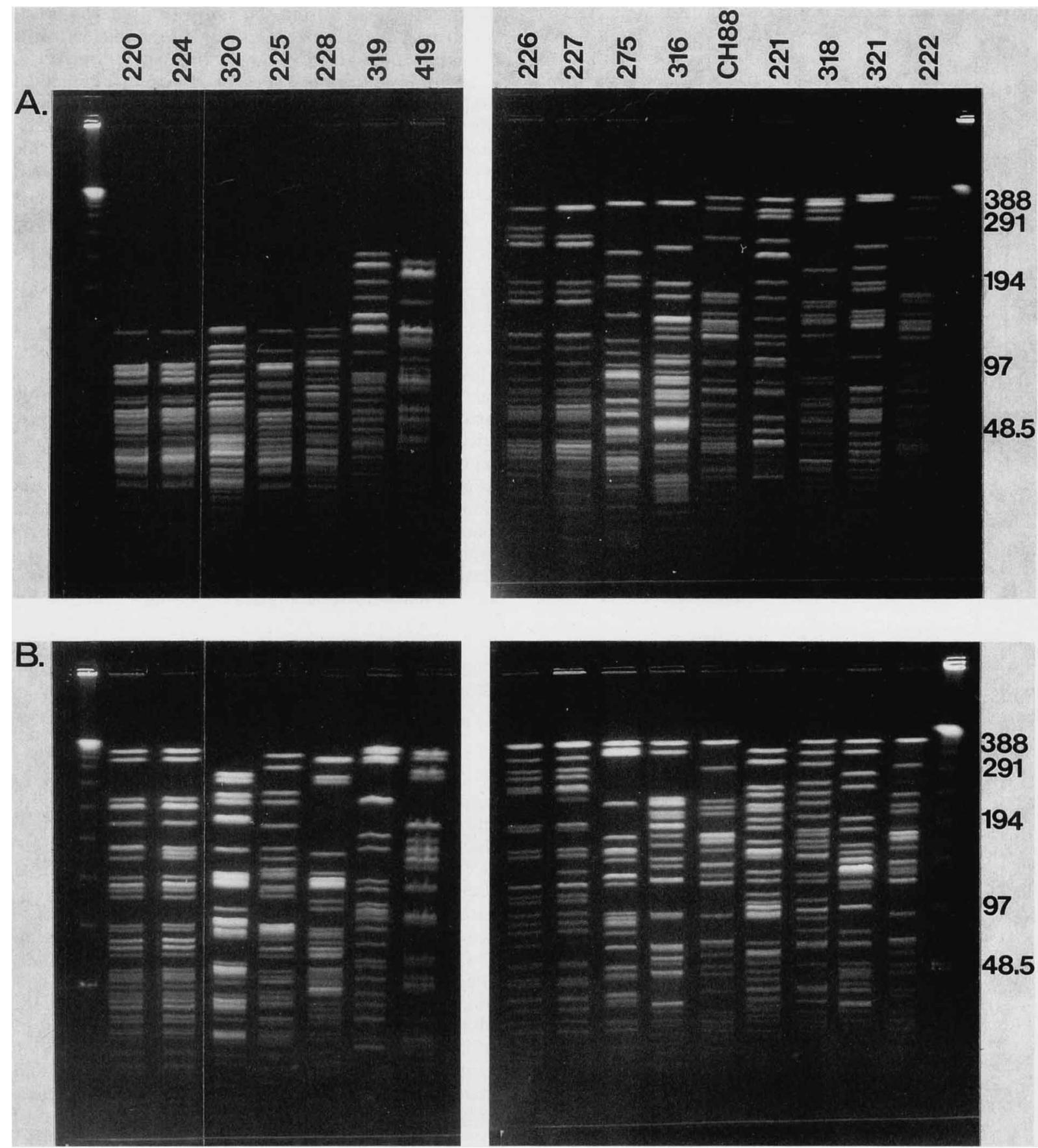

FIG. 1. Separation of $X b a \mathrm{I}(\mathrm{A})$ and SpeI (B) digests of $P$. stutzeri chromosomes by contour-clamped homogeneous electric field gel electrophoresis. Fragments were resolved by using a ramped pulse time of 1 to $25 \mathrm{~s}$ for $23 \mathrm{~h}$ (1.3\% agarose). The sizes of comigrating lambda concatemers (in kilobase pairs) are indicated on the right.

profiles were sufficiently similar that the strains could be considered members of the same taxospecies.

Fatty acid analysis. Under standardized growth conditions all of the strains produced qualitatively similar FAME profiles. The fatty acids common to all isolates included saturated dodecanoic $\left(\mathrm{C}_{12: 0}\right)$, tetradecanoic $\left(\mathrm{C}_{14: 0}\right)$, hexadecanoic $\left(\mathrm{C}_{16: 0}\right)$, and octadecanoic $\left(\mathrm{C}_{18: 0}\right)$ acids, unsaturated 3-hydroxydecanoic $\left(\mathrm{C}_{10: 0} \quad 3 \mathrm{OH}\right), 3$-hydroxydodecanoic $\left(\mathrm{C}_{12: 0} 3 \mathrm{OH}\right)$, cis-9 hexadecanoic $\left(\mathrm{C}_{16: 1}\right.$ cis-9), and 15-meth- ylhexadecanoic $\left(\mathrm{C}_{17: 0}\right.$ iso) acids, and an isomer of octadecanoic $\left(C_{18: 1}\right)$ acid (summed feature 7$)$ (Table 3 ). Three fatty acids, $\mathrm{C}_{16: 1}$ cis-9, $\mathrm{C}_{16: 0}$, and an isomer of $\mathrm{C}_{18: 1}$ (summed feature 7 ), accounted for more than $83 \%$ of the total fatty acid composition.

The results of three determinations showed that the fatty acid profiles were highly reproducible, both quantitatively and qualitatively. The coefficient of variation ([standard deviation/mean] $\times 100$ ) for each fatty acid that accounted for 

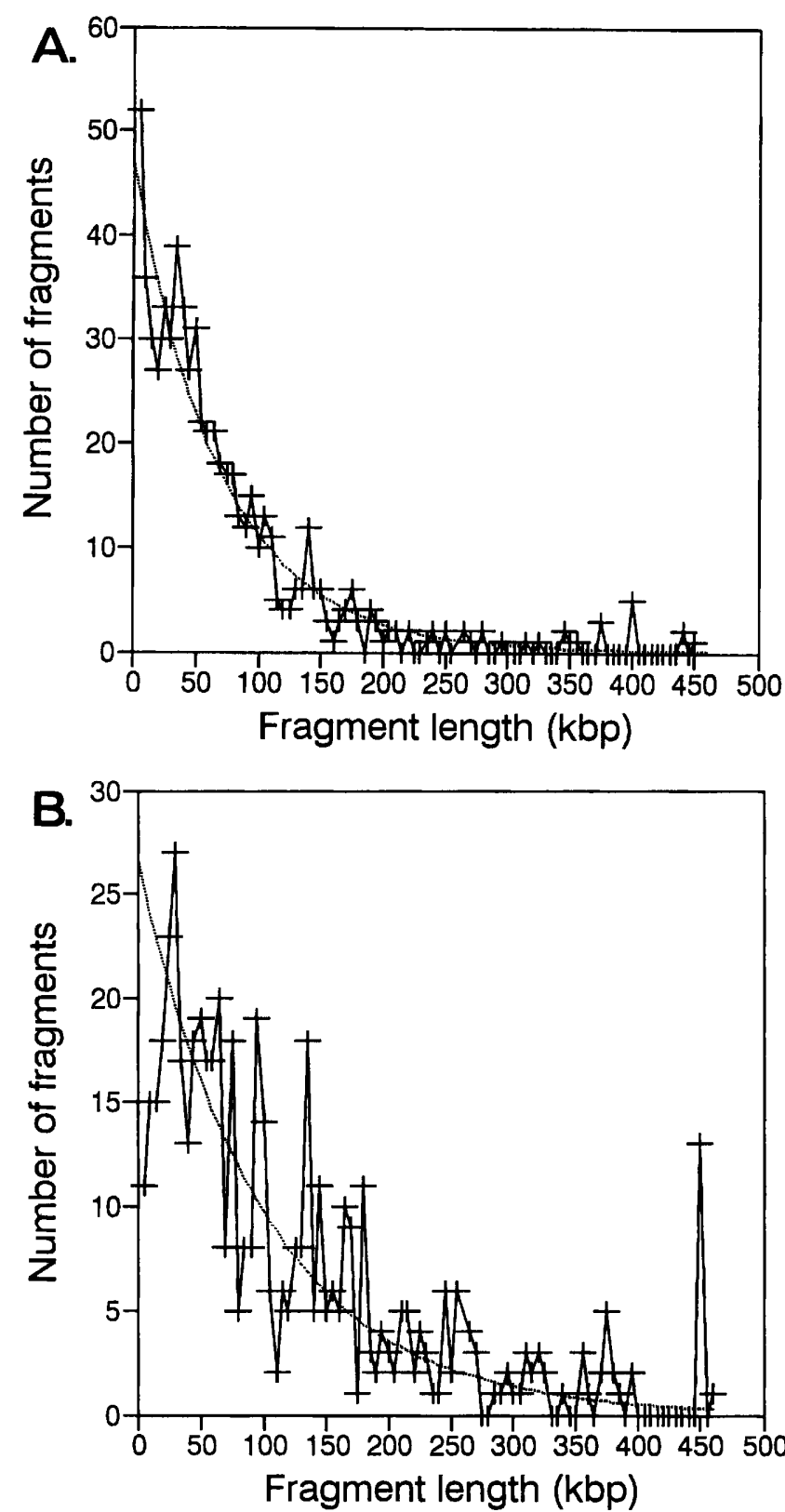

FIG. 2. Distribution of $X b a I$ (A) and SpeI (B) restriction fragments from $16 P$. stutzeri strains. The theoretical distribution of fragments (assuming a random distribution of enzyme recognition sites) is shown by the dotted line.

more than $2.58 \%$ of the total fatty acid content in each cluster was less than $10 \%$.

The dendrogram in Fig. 4 revealed a single cluster at a distance of 9.0 Euclids, but at a distance of 3.0 Euclids three clusters were evident. The differences between these clusters were largely accounted for by small differences in the relative amounts of fatty acids shown in Table 3. Four strains (strains 222, 316, 318 and 275) were not assigned to any cluster. Cluster I contained strains that had low $\mathrm{G}+\mathrm{C}$ contents and included four strains isolated from clinical material in Copenhagen, Denmark, and one strain isolated from clinical material in Paris, France. The remainder of the clinical strains were distributed throughout the dendrogram, and strains isolated from the soil were found in clusters II and III (strain 275 was not assigned to a cluster). All of the strains except strain 318 were identified as $P$. stutzeri, and all of the strains except strains 275,319 , and 419 received high similarity index values $(>0.7)$ when they were compared with the Microbial Indentification System aerobe library. Strains 275,319 , and 419 received similarity index values of less than 0.3. Strain 318 was identified as Pseudomonas mendocina with a high degree of certainty (similarity index, 0.83 ), although in terms of general phenotypic properties this strain resembles $P$. stutzeri more closely (18).

Numerical analysis of substrate utilization profiles. The detailed substrate utilization data of Palleroni et al. (18) for all $16 P$. stutzeri strains were analyzed by using numerical methods (Fig. 5). All of the strains clustered in a single group at a distance of 5.0 Euclids, but three clusters were observed below 3.2 Euclids. Five strains (strains 316, 228, 319, 419, and 275 ) were not assigned to any cluster. There was no apparent correlation with the clusters obtained by macrorestriction fragment analysis or FAME analysis, but there was some correlation with geographical source. Substrate utilization cluster III highlights the differences between the results of clustering based on substrate utilization and the results of clustering based on genomic measurements. Strains 320 and 321 were both isolated from clinical specimens at the Institut Pasteur, Paris, France, and produced very similar substrate utilization profiles, differing only in their ability to utilize mucate and valerate (18); however, on the basis of macrorestriction fragment analysis they were assigned to separate groups, a reflection of their markedly different $\mathrm{G}+\mathrm{C}$ contents (Table 1) (18).

\section{DISCUSSION}

Application of modern analytical methods to the taxonomy of $P$. stutzeri has confirmed previous results $(11,22)$ which suggested that subdivision of $P$. stutzeri is possible on the basis of genomic characteristics. The results of a macrorestriction fragment analysis supported the hypothesis that there is a distinction between strains having low $\mathrm{G}+\mathrm{C}$ contents (approximately $62 \mathrm{~mol}$ ) and strains having high $\mathrm{G}+\mathrm{C}$ contents ( 64 to $65 \mathrm{~mol} \%)$. This distinction was also reflected in the results of the FAME analysis and is significant because correlations between phenotypic and genotypic subdivisions have been observed only rarely for $P$. stutzeri strains. While our results are a step toward unravelling the taxonomy of this heterogeneous group, we feel that it is premature to recommend subdivision of the species, especially given the poor correlation with substrate utilization properties, and agree that "useful specific division" is still not feasible $(18,22)$. Future use of alternative methods of phenotypic analysis, such as gas chromatography of FAMEs and pyrolysis-mass spectroscopy, may eventually result in a meaningful division. A sequence analysis of conserved "housekeeping" genes may also yield valuable information concerning the taxonomy and phylogeny of these strains.

While the observed distinction between strains having high and low $\mathrm{G}+\mathrm{C}$ contents is encouraging, our sample of both clinical and nonclinical strains included examples which reflect the two extremes of the $P$. stutzeri $\mathrm{G}+\mathrm{C}$ content spectrum, and thus distinctions may be less obvious when strains having $\mathrm{G}+\mathrm{C}$ contents of approximately 63 $\mathrm{mol} \% \mathrm{G}+\mathrm{C}$ are included. Nevertheless, the observed differences between the two groups indicate the value of the methods used in this study and suggest that a more compre- 
TABLE 2. Pairwise comparisons of the cumulative macrorestriction fragment patterns of $P$. stutzeri strains

\begin{tabular}{|c|c|c|c|c|c|c|c|c|c|c|c|c|c|c|c|}
\hline \multirow{2}{*}{ Strain } & \multicolumn{15}{|c|}{ Level of significance ( $P$ value) for comparison with $\operatorname{strain}^{a}$ : } \\
\hline & 220 & 224 & 320 & 225 & 228 & 319 & 419 & 222 & 321 & 318 & 221 & $\mathrm{CH} 88$ & 316 & 275 & 227 \\
\hline 224 & 0.001 & & & & & & & & & & & & & & \\
\hline 320 & 0.01 & 0.05 & & & & & & & & & & & & & \\
\hline 225 & 0.001 & 0.001 & 0.001 & & & & & & & & & & & & \\
\hline 228 & 0.001 & 0.001 & 0.001 & 0.01 & & & & & & & & & & & \\
\hline 319 & 0.05 & 0.05 & - & - & - & & & & & & & & & & \\
\hline 419 & 0.05 & 0.05 & - & - & - & 0.01 & & & & & & & & & \\
\hline 222 & - & - & - & - & - & - & 0.05 & & & & & & & & \\
\hline 321 & 0.01 & 0.01 & - & 0.05 & - & - & - & - & & & & & & & \\
\hline 318 & - & - & - & - & - & - & 0.05 & 0.05 & 0.01 & & & & & & \\
\hline 221 & - & - & - & - & - & - & - & - & - & - & & & & & \\
\hline $\mathrm{CH} 88$ & 0.05 & 0.05 & - & 0.01 & - & - & - & 0.001 & 0.05 & 0.01 & 0.01 & & & & \\
\hline 316 & 0.001 & 0.001 & - & - & 0.01 & 0.01 & - & 0.01 & 0.01 & 0.01 & 0.01 & 0.05 & & & \\
\hline 275 & 0.05 & 0.05 & - & - & - & 0.05 & 0.05 & - & - & - & 0.05 & 0.05 & 0.01 & & \\
\hline 227 & - & - & 0.01 & - & - & - & - & - & 0.01 & - & 0.05 & - & - & - & \\
\hline 226 & - & - & 0.05 & - & - & - & - & - & 0.01 & - & 0.05 & - & 0.05 & - & 0.001 \\
\hline
\end{tabular}

${ }^{a}$ Levels of significance for pairs of strains whose restriction fragment patterns are more similar than those expected by chance. - pairs of strains whose restriction fragment patterns are no more or no less similar than those expected by chance (that is, the $S_{D}$ values are with in the $95 \%$ confidence interval).

hensive taxonomic study of this species by using FAME and macrorestriction fragment analyses would be worthwhile.

Previous work showed that growth at a high temperature was one of the few phenotypic properties which correlated with $\mathrm{G}+\mathrm{C}$ content (24), and given the importance of this result, growth temperature experiments were repeated. Our results were identical to those reported previously except for strain 319 , which did not grow at $42^{\circ} \mathrm{C}$ (data not shown). Thus, except for strains 275,319 , and 419 , the ability to grow at $43^{\circ} \mathrm{C}$ correlated with macrorestriction fragment groups;

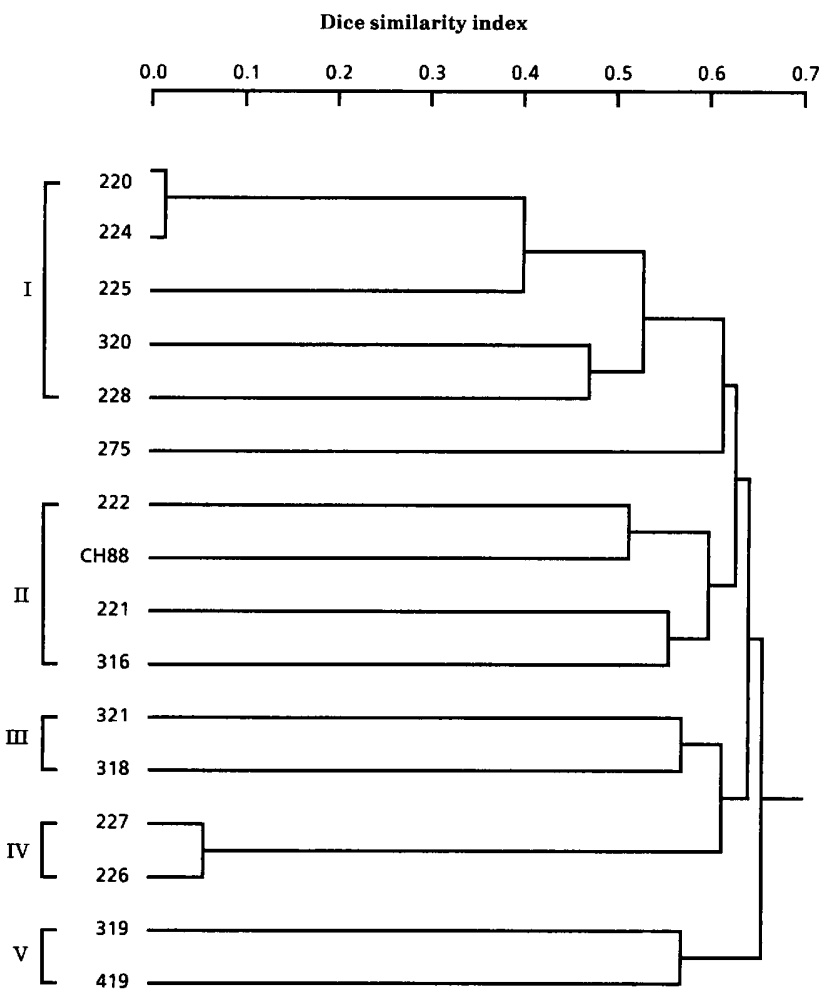

FIG. 3. Dendrogram of $P$. stutzeri strains generated by cluster analysis of macrorestriction fragment patterns with the UPGMA. strains assigned to macrorestriction fragment cluster I and FAME cluster I (low-G+C-content strains) were not able to grow at $43^{\circ} \mathrm{C}$. This result is also consistent with the results of Rosselló et al. (22), although these authors found that growth at 40 and $42^{\circ} \mathrm{C}$ is useful for distinguishing between genomic groups. Interestingly, strains 275,319 , and 419 were least similar to the other strains in all of the characteristics examined in this study; strain 419 is recognized as being highly atypical in both genotypic and phenotypic properties $(22,24)$.

A substantial degree of correlation was observed between clusters obtained on the basis of the results of the macrorestriction fragment analysis and the genomovar groups of Rosselló et al. (22), which were based on the results of DNA-DNA hybridization studies. Among the strains examined by Rosselló et al. (22) were 10 strains included in this study. Macrorestriction fragment cluster I contained strains $220,224,225$, and 228 , all of which were assigned to genomovar 2 (22). Macrorestriction fragment clusters II and IV (which differ by no more than $1.5 \%$ on the $S_{D}$ similarity index [Fig. 3]) each contained two strains (strains 221 and 222 and strains 226 and 227, respectively) which were assigned to genomovar 1. Macrorestriction fragment cluster

TABLE 3. Fatty acid compositions of $P$. stutzeri strains

\begin{tabular}{lccr}
\hline \multirow{2}{*}{ Fatty acid } & \multicolumn{3}{c}{$\%$ in $^{a}:$} \\
\cline { 2 - 4 } & $\begin{array}{c}\text { Cluster I } \\
(n=6)^{b}\end{array}$ & $\begin{array}{c}\text { Cluster II } \\
(n=5)\end{array}$ & $\begin{array}{c}\text { Cluster III } \\
(n=2)\end{array}$ \\
\hline $\mathrm{C}_{10: 0}$ 3OH & $2.58(10.0)^{c}$ & $2.33(9.0)$ & $2.33(3.0)$ \\
$\mathrm{C}_{12: 0}$ & $8.03(4.0)$ & $7.68(2.0)$ & $8.38(3.0)$ \\
$\mathrm{C}_{12: 0}$ 3OH & $3.31(5.0)$ & $3.14(6.0)$ & $3.31(6.0)$ \\
$\mathrm{C}_{14: 0}$ & $0.64(9.0)$ & $1.02(1.2)$ & $0.99(4.0)$ \\
$\mathrm{C}_{16: 1}$ cis-9 & $28.43(3.0)$ & $26.20(3.0)$ & $32.72(1.0)$ \\
$\mathrm{C}_{16: 0}$ & $16.18(4.0)$ & $18.80(5.0)$ & $18.78(4.0)$ \\
$\mathrm{C}_{17: 0}$ iso & $0.52(22.0)$ & $0.39(1.6)$ & $0.43(1.9)$ \\
$\mathrm{C}_{18: 0}$ & $0.59(9.0)$ & $0.48(4.2)$ & $0.35(5.0)$ \\
Summed feature 7 & $38.93(2.0)$ & $39.18(2.0)$ & $31.93(2.0)$ \\
\hline
\end{tabular}

${ }^{a}$ Mean fatty acid compositions of isolates in clusters I through III (each strain was analyzed three times).

${ }^{b} n$ is the number of strains examined.

$c$ The numbers in parentheses are coefficients of variation. 


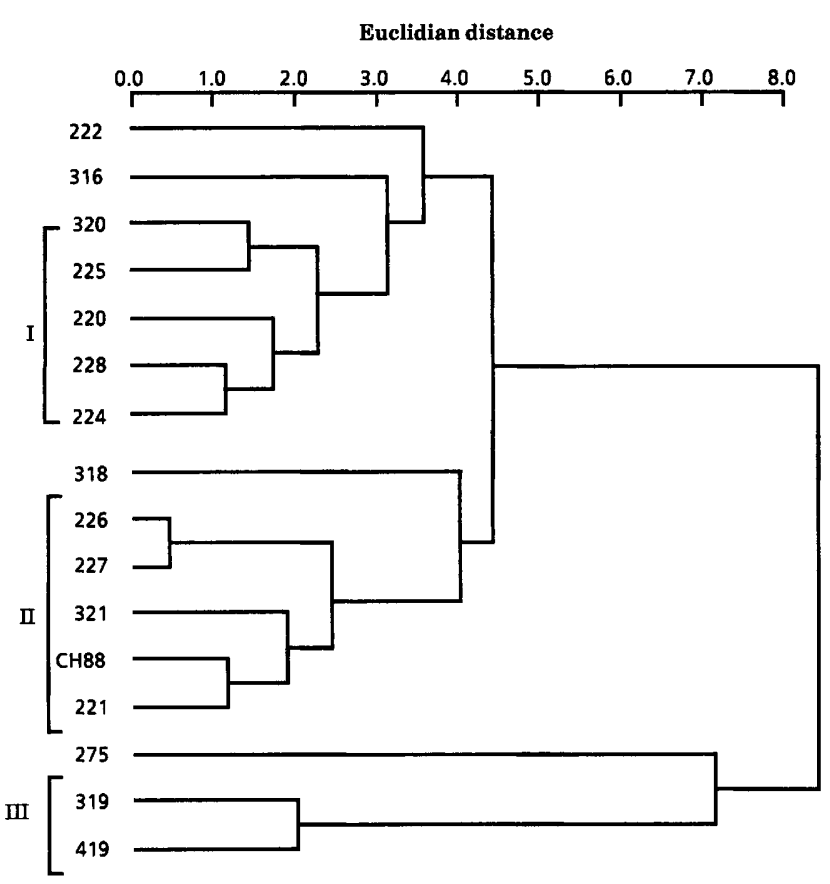

FIG. 4. Dendrogram of $P$. stutzeri strains generated by cluster analysis of FAME profiles with the UPGMA.

II also contained strain CH88, which Rosselló et al. (22) assigned to genomovar 3. Macrorestriction fragment cluster $\mathrm{V}$ contained atypical strain $419(22,24)$, which was assigned to genomovar 7 (22). Thus, macrorestriction fragment cluster I appears to correspond to genomovar 2 (and group I of Palleroni et al. [18]), macrorestriction fragment clusters II and IV correspond approximately to genomovar 1 , and macrorestriction fragment cluster $\mathrm{V}$ corresponds to genomovar 7.

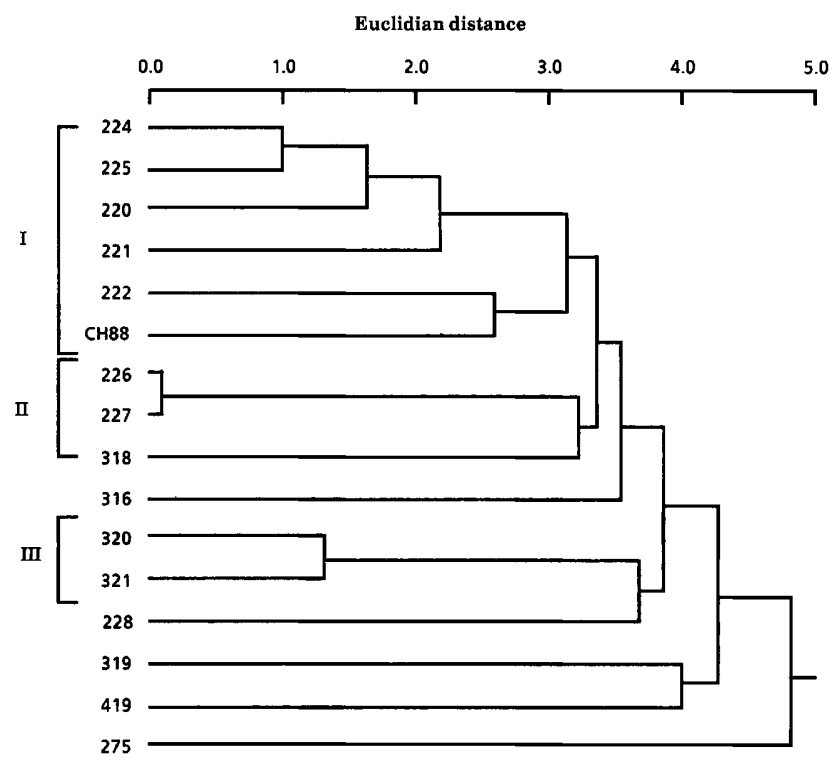

FIG. 5. Dendrogram of $P$. stutzeri strains generated by cluster analysis of the substrate utilization profiles obtained from the study of Palleroni et al. (18) with the UPGMA.
A similar degree of correlation was observed between the genomovar groups and the FAME clusters. FAME cluster I contained the low-G+C-content strains corresponding to genomovar 2, and FAME cluster II contained three strains belonging to genomovar 1 but also contained strain $\mathrm{CH} 88$ (genomovar 3); strain 222 (genomovar 2) was not located in any cluster. FAME cluster III, which contained strain 419 (genomovar 7), was well separated from the other clusters.

In addition to its taxonomic value, macrorestriction fingerprinting provided useful information concerning the genetic structure of and variation among $P$. stutzeri strains. The nonrandom distribution of both $X b a I$ and SpeI sites (especially SpeI, sites) within the $P$. stutzeri genome suggests that rare palindromes play a role in determining chromosome structure (9). A similar nonrandom distribution of SpeI fragments was observed in Pseudomonas aeruginosa (6) and also in a population of 30 Pseudomonas fluorescens strains isolated from the phyllosphere of sugar beets (27). This appears to be a common and perhaps significant feature of Pseudomonas chromosomes which warrants further investigation.

Macrorestriction fragment analysis also revealed two clear examples of evolutionary divergence among the 16 strains; strains 220 and 224 obviously shared common ancestory, as did strains 226 and 227. A statistical analysis indicated that strains 225,228 , and 320 also belonged to the same lineage as strains 220 and 224 (macrorestriction fragment cluster I). It is therefore interesting that strains 220,224 , and 225 were isolated in 1956 from different clinical specimens at the Statens Seruminstitut, Copenhagen, Denmark, while strain 228 was isolated in 1957 (16a). While strain 320 apparently is a member of the same lineage, it was isolated from clinical material in Paris, France (the year of isolation is not known). Strains 226 and 227 were both isolated from clinical material at the Statens Seruminstitut in 1957. The occurrence of two divergent lineages within such a small collection of strains suggests that divergence among $P$. stutzeri strains may be common.

With the exception of strains 220 and 224 and strains 226 and 227 , the level of variation among macrorestriction profiles was considerable given the phenotypic similarity (both FAME and substrate utilization) among strains. Even between strains that exhibit substantial levels of phenotypic similarity (for example, strains 220 and 225 , which produced identical substrate utilization profiles for 77 of 80 different substrates [18] and were both members of FAME cluster I) marked differences between macrorestriction profiles were observed. While the calculated level of similarity between the macrorestriction profiles of strains 220 and $225(60 \%)$ was high enough to indicate a highly significant degree of relatedness $(P<0.001)$, the differences in genome structure suggest the possibility that recombination (either intragenomic or intergenomic) may have been a significant factor in generating the heterogeneity observed. Indeed, the heterogeneity inherent at all levels in $P$. stutzeri may reflect two significant factors: (i) niche-specific selection and (ii) an ability to take up and incorporate foreign DNA $(1 \mathrm{a}, 21)$. The diverse variety of niches occupied by $P$. stutzeri isolates and the known natural competence of $P$. stutzeri $(1,10,25)$ make both factors distinct possibilities.

\section{ACKNOWLEDGMENTS}

We are grateful to J. S. Fenlon for statistical advice and M. J. Bailey for helpful discussions.

I.P.T. receives funds from the Department of the Environment. 


\section{REFERENCES}

1. Carlson, C. A., L. S. Pierson, J. J. Rosen, and J. L. Ingraham. 1983. Pseudomonas stutzeri and related species undergo natural transformation. J. Bacteriol. 153:93-99.

1a.Cohan, F. M. Submitted for publication.

2. Cuypers, H., and W. G. Zumft. 1992. Regulatory components of the denitrification gene cluster of Pseudomonas stutzeri, p. 188-197. In E. Galli, S. Silver, and B. Witholt (ed.), Pseudomonas: molecular biology and biotechnology. American Society for Microbiology, Washington, D.C.

3. Daughton, C. G., and W. C. Evans. 1977. Parathion utilization by bacterial symbionts in a chemostat. Appl. Environ. Microbiol. 34:174-184.

4. Giovannetti, L., S. Ventura, M. Bazzicalupo, R. Fani, and R. Materassi. 1990. DNA restriction fingerprint analysis of the soil bacterium Azospirillum. J. Gen. Microbiol. 136:1161-1166.

5. Gower, J. C. 1966. Some distance properties of latent root and vector methods used in multivariate analysis. Biometrika 53: 325-338.

6. Grothues, D., and B. Tümmler. 1991. New approaches in genome analysis by pulsed-field gel electrophoresis: application to the analysis of Pseudomonas species. Mol. Microbiol. 5:2763-2776.

7. Hayashida, S., S. Tanaka, Y. Teramoto, N. Nanari, S. Yoshino, and K. Furukawa. 1991. Isolation of anti-algal Pseudomonas stutzeri strains and their lethal activity for Chattonella antiqua. Agric. Biol. Chem. 55:787-790.

8. Holmes, B. 1986. Identification and distribution of Pseudomonas stutzeri in clinical material. J. Appl. Bacteriol. 60:401-411.

9. Krawiec, S., and M. Riley. 1990. Organization of the bacterial chromosome. Microbiol. Rev. 54:502-539.

10. Lorenz, M. G., and W. Wackernagel. 1991. High frequency of natural genetic transformation of Pseudomonas stutzeri in soil extract supplemented with a carbon-energy and phosphorus source. Appl. Environ. Microbiol. 57:1246-1251.

11. Mandel, M. 1966. Deoxyribonucleic acid base composition in the genus Pseudomonas. J. Gen. Microbiol. 43:273-292.

12. McClelland, M., J. Hanish, M. Nelson, and Y. Patel. 1988. KGB: a single buffer for all restriction endonucleases. Nucleic Acids Res. 16:364.

13. McClelland, M., R. Jones, Y. Patel, and M. Nelson. 1987. Restriction endonucleases for pulsed field mapping of bacterial genomes. Nucleic Acids Res. 15:5985-6005.

14. Miller, L., and T. Berger. 1985. Bacteria identification by gas chromatography of whole cell fatty acids. Hewlett-Packard application note 228-41. Hewlett-Packard Co., Avondale, Pa.

15. Nei, M., and W.-H. Li. 1979. Mathematical model for studying genetic variation in terms of restriction endonucleases. Proc. Natl. Acad. Sci. USA 76:5269-5273.

16. Palleroni, N. J. 1984. Pseudomonadaceae, p. 141-199. In N. R. Kreig and J. G. Holt (ed.), Bergey's manual of systematic bacteriology, vol. 1. The Williams \& Wilkins Co., Baltimore.

16a.Palleroni, N. J. Unpublished data.

17. Palleroni, N. J., and M. Doudoroff. 1972. Some properties and taxonomic subdivisions of the genus Pseudomonas. Annu. Rev. Phytopathol. 10:73-100.

18. Palleroni, N. J., M. Doudoroff, R. Y. Stanier, R. E. Solánes, and M. Mandel. 1970. Taxonomy of the aerobic pseudomonads: the properties of the Pseudomonas stutzeri group. J. Gen. Microbiol. 60:215-231.

19. Palleroni, N. J., R. Kunisawa, R. Contopoulou, and M. Doudoroff. 1973. Nucleic acid homologies in the genus Pseudomonas. Int. J. Sys. Bacteriol. 23:333-339.

20. Rainey, P. B., C. L. Brodey, and K. Johnstone. 1993. Identification of a gene cluster encoding three high molecular weight proteins which is required for synthesis of tolaasin by the mushroom pathogen Pseudomonas tolaasii. Mol. Microbiol. 8:643-652.

21. Reeves, P. R. 1992. Variation in O-antigens, niche-specific selection and bacterial populations. FEMS Microbiol. Lett. 100:509-516.

22. Rosselló, R., E. Garcia-Valdes, J. Lalucat, and J. Ursing. 1991. Genotypic and phenotypic diversity of Pseudomonas stutzeri. Syst. Appl. Microbiol. 14:150-157.

23. Sneath, P. H. A., and R. R. Sokal. 1973. Numerical taxonomy: the principles and practice of numerical classification. W. $\mathrm{H}$. Freeman, San Francisco.

24. Stanier, R. Y., N. J. Palleroni, and M. Doudoroff. 1966. The aerobic pseudomonads: a taxonomic study. J. Gen. Microbiol. 43:159-271.

25. Stewart, G. J., and C. D. Sinigalliano. 1991. Exchange of chromosomal markers by natural transformation between the soil isolate, Pseudomonas stutzeri JM300, and the marine isolate, $P$. stutzeri strain Zobell. Antonie van Leeuwenhoek J. Microbiol. 59:19-25.

26. Suwanto, A., and S. Kaplan. 1989. Physical and genetic mapping of the Rhodobacter sphaeroides 2.4.1 genome: genome size, fragment identification, and gene localization. J. Bacteriol. 171:5840-5849.

27. Thompson, I. P., and P. B. Rainey. Submitted for publication.

28. Woo, G. J., and J. D. McCord. 1991. Maltotetraose production using Pseudomonas stutzeri exo-alpha-amykase in a membrane recycle bioreactor. J. Food Sci. 56:1019-1023. 